\title{
Ultra-low Power Single-walled Carbon Nanotube Based Chemical Sensors
}

\author{
K. Chikkadi ${ }^{1}$, C. Roman ${ }^{1}, \underline{\text { C. Hierold }}{ }^{1}$ \\ ${ }^{1}$ ETH Zurich, Micro and Nanosystems, Zurich, Switzerland \\ christofer.hierold@micro.mavt.ethz.ch
}

\begin{abstract}
:
In the last decade, Single-walled Carbon Nanotubes (SWNTs) have emerged as potential functional materials in next-generation chemical sensors due to their exceptional electronic and chemical properties. In this review, we summarize the progress that has been made in the last few years in the field of carbon nanotube chemical sensors. When a single SWNT is integrated as the channel in a carbon nanotube field-effect transistor (CNFET), ultra-low power operation down to $0.01 \mu \mathrm{W}$ and a detection limit of $50 \mathrm{ppb}$ (parts per billion) $\mathrm{NO}_{2}$ at room temperature has been achieved. Hysteresis suppression through pulsed gate sweep strategies and sensor recovery by heating and UV-exposure has been demonstrated. In case of functionalized CNT-network sensors, detection limits as low as 100 ppt (parts per trillion) has been achieved.
\end{abstract}

Key words: Carbon nanotubes, chemical sensors, low power, hysteresis, sensitivity, chem-FET

\section{Introduction}

Ever since J. Kong et al. [1] demonstrated the chemical detection of $\mathrm{NO}_{2}$ and $\mathrm{NH}_{3}$ with CNFETs, SWNT-based gas sensors have been studied extensively. For a SWNT, all the atoms contributing to electronic transport are at the surface, and available for interaction with the environment. The carbon atoms in a CNT are linked by strong $\mathrm{sp}^{2}$ bonds, making them chemically inert. However, non-covalent interactions at the surface are possible, and the sensitivity of SWNTs to different chemical species, including $\mathrm{NO}_{2}, \mathrm{O}_{2}, \mathrm{NH}_{3}$, polymers and biomolecules has been shown [2].

Depending on the number of CNTs present as sensing material in the channel, the devices are classified as mat-sensors or individual SWNT sensors. In mat-sensors, multiple CNTs are assembled between electrodes. This increases the area available for interaction with the analyte, but it also increases the power consumption. Due to the presence of intersections and crossings between CNTs in the channel, the transduction mechanism in these devices is also complex to model. Besides low power consumption, Individual SWNT sensors are expected to be easier to model and to provide a platform for understanding the transduction mechanisms and device physics in CNT-based sensors.

Despite their high potential and promise, the initial demonstrations of SWNT-based sensors also exhibited several issues which hindered their application in practical situations, such as controlled integration of CNTs into microstructures [3], gate hysteresis [4], sensing at the metal-CNT contacts [5] and high $1 / f$ noise [6]. Recently, novel measurement techniques and improvements in sensor design and fabrication have led to devices with improved performance with respect to sensitivity, detection limit and long-term stability.

\section{Recent progress in SWNT-based chemical sensors}

\section{Hysteresis}

The first report on CNT sensors [1] showed sensitivity of the electrical characteristics of a single SWNT connected between two $\mathrm{Ni} / \mathrm{Au}$ electrodes to $\mathrm{NO}_{2}$ and $\mathrm{NH}_{3}$. They found that upon exposure to the analyte, there was a shift of the threshold voltage in the transfer characteristics of the FET (Fig. 1). In this paper, no gate hysteresis is reported since the gate voltage was swept only in one direction.

Helbling et al. in 2008 [7] studied the $\mathrm{NO}_{2}$ sensing behavior of suspended CNFETs. Their measurements indicate a large gate hysteresis in the transfer characteristics of chem-FET measurements. On devices with an open channel, it has been shown that water molecules surrounding the CNT have a large influence on gate hysteresis $[8,9]$. However, in passivated CNFETs the gate hysteresis has been attributed 
to traps in the dielectric environment surrounding of the CNT channel, which at large gate voltages become charged and discharged under large electrostatic field created by the CNT [10]. In both cases, the electrical environment and history of the sensor appears to have a strong influence on the resulting sensor output. Therefore, a method for defining the sensor baseline for eventual calibration is necessary.

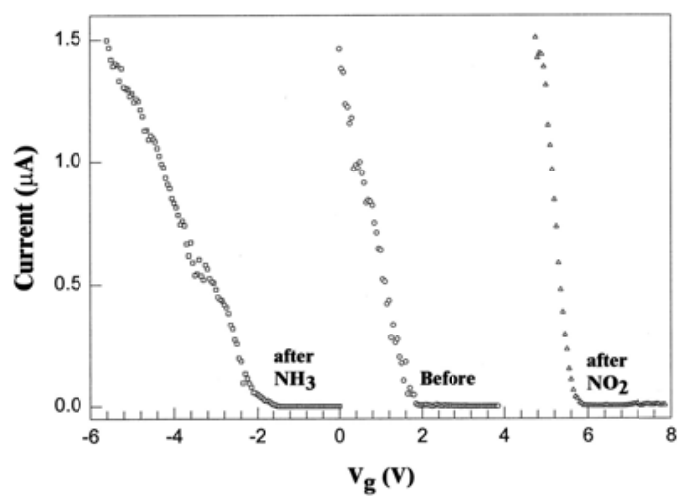

Fig. 1 The first gas sensor measurements reported by Kong et al. [1] with a single carbon nanotube connected between metal electrodes in a back-gated FET configuration. The threshold voltage shifts in opposite directions for $\mathrm{NO}_{2}$ and $\mathrm{NH}_{3}$.It is suggested that $\mathrm{NO}_{2}$ being an electron-withdrawing species, p-dopes the CNT and causes a right-shift of the threshold, while $\mathrm{NH}_{3}$ has the opposite effect. Reprinted from Kong et al. [1] @ 2000 American Association for the Advancement of Science

Lin et al. [9] - for general CNFETs -, and later Mattmann et al. [11-13] - for CNFET gas sensors - have demonstrated the use of pulsed gate measurement techniques to eliminate the gate hysteresis. Instead of a quasi-static linear gate sweep, applying alternating positive and negative pulses on the gate was shown to suppress the hysteresis (Fig. 2). With this technique, they were able to detect $50 \mathrm{ppb}$ of $\mathrm{NO}_{2}$. It was also shown that this technique could be used to extend the measurement range and erase the electrical history of the sensor [14].

More recently, Muoth et al. demonstrated CNFETs with ultra-clean CNTs which could be fabricated by either shadow-mask lithography [15] or post-deposition assembly techniques [16], which resulted in zero hysteresis in ambient conditions. Such devices offer another pathway for fabricating hysteresis-free carbon nanotube chemical sensors.

\section{Sensor recovery}

In [1], Kong et al. demonstrated the possibility to recover the original sensor signal after exposure, either by desorption at ambient temperatures in $12 \mathrm{~h}$ or at $200{ }^{\circ} \mathrm{C}$ for $1 \mathrm{~h}$. This has since been confirmed by Mattmann et al [13] by heating to $110{ }^{\circ} \mathrm{C}$. Adapting a different approach, Li et al.
[17] have demonstrated the use of UV light to desorb analytes from the sensor. Use of electrical impulses to refresh the sensor has also been shown by Chang et al [18]. However, the use of electrical impulses can also affect the threshold voltage of the sensor as explained in [13]. Therefore, further investigations are required to confirm that electrical impulses could affect sensor recovery.
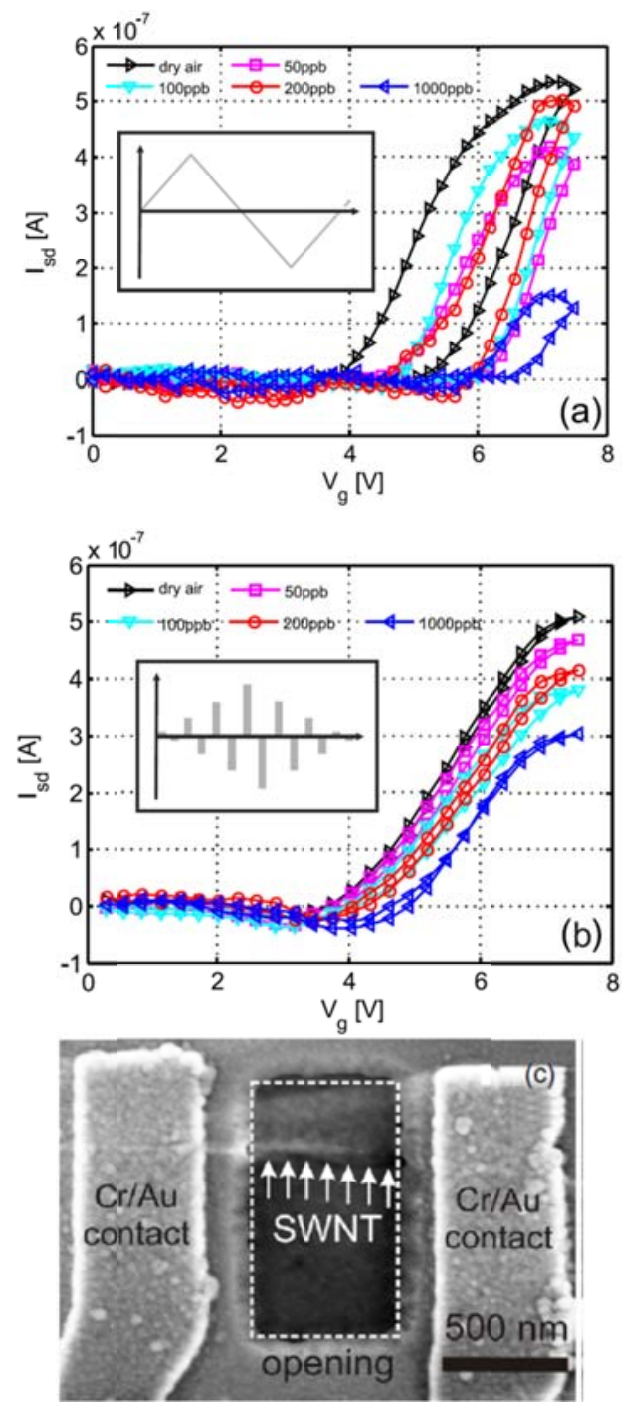

Fig. 2: CNFET transfer characteristics for (a) continuous gate sweep and (b) pulsed sweep at different $\mathrm{NO}_{2}$ concentrations as shown by Mattmann et al. (c) an SEM image of the gas sensor used in the study. An enhancement in the detection limit as well as the range of the sensor is seen. Figures adapted from [13]. (C) 2010 IOP publishing.

\section{Sensing mechanism}

The mechanism of $\mathrm{NO}_{2}$ sensing is currently debated with effects attributed to both the contacts and the channel. Several sensing mechanisms, including charge transfer and electrostatic gating at the CNT channel [1], 
changes in the Schottky Barrier (SB) height and width in the contact regions [19] have been suggested.

Heller et al. investigated CNFETs for biosensing [5] and suggest that metal-SWNT contacts could respond unreliably to certain analytes. Furthermore, while the CNT is considered chemically inert, the reactivity of the contact metal to certain analytes cannot be excluded. Therefore, a device structure with the contacts isolated from the analyte is desirable. Mattmann et al. [13] proposed a partially passivated CNFET where 40 $\mathrm{nm}$ of $\mathrm{Al}_{2} \mathrm{O}_{3}$ deposited by atomic layer deposition was used to passivate the contacts, while leaving the channel open to the analyte. They also demonstrate that a control structure with a closed channel shows no response to $\mathrm{NO}_{2}$ in the same experiment, demonstrating that passivation is effective.

However, investigations on CNT mat-sensors and SWNT-FETs passivated with polymer layers have discussed sensing mechanisms at the contacts [20]. It is argued that the CNT-metal work function is modified by gas adsorption near the contacts, which leads to changes in the ellectric characteristics. However, as discussed by Heller et al. [5], such a mechanism would actually lead to a so-called 'rotation' of the transfer characteristic rather than the rigid shift of the curve as is usually observed.

The choice of analyte, carbon nanotube defect density [21] and contact metal could also affect the sensing mechanism, and multiple mechanisms could exist in some device configurations.

\section{Detection limit and selectivity in CNFETs}

Kong et al. showed in 2001 [22] the possibility of decorating CNFETs with a non-continuous $\mathrm{Pd}$ layer to enhance sensitivity to hydrogen. They demonstrated response times in the order of 5 $10 \mathrm{~s}$ and a recovery time of about $400 \mathrm{~s}$ under ambient conditions. The lowest concentration tested in the study was $4 \mathrm{ppm}$. With CNT mat sensors coated with PEI (polyethyleneimine), a detection limit down to 100 ppt to $\mathrm{NO}_{2}$ has been demonstrated [23] (Fig. 3). Further techniques to improve the selectivity involve arrays of CNFETs which are decorated with different functional materials or have contact metals with different work functions. Depending on the analyte, the responses on different functional coatings may be different, resulting in unique 'signatures' for the analyte. Such approaches with CNT sensors have been outlined in [20]. Currently, mechanisms involved in specific interactions between the analyte and functional materials are not completely understood, and a deeper understanding of these mechanisms is required for a sound basis for the design of these functional materials to enhance the selectivity of CNFET sensors.

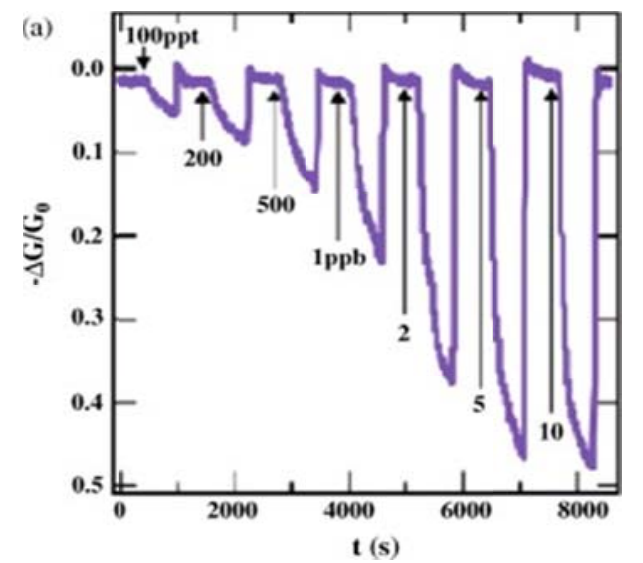

Fig. 3: Qi et al. [23] demonstrated PEl-functionalized mat-CNFET used for sensing $\mathrm{NO}_{2}$. The minimum concentration detected in this study was as low as 100 ppt, and saturated at $3 \mathrm{ppb}$. Reprinted with permission from Qi et al. [23]. (c) 2003 American Chemical Society.

\section{Sensitivity and noise performance}

Noise in CNFETs is a well-documented problem $[24,25]$. It is observed that CNFETs exhibit high $1 / f$ noise, which has been attributed to the low number of carriers in the CNT channel, as well as the high sensitivity of the CNT to its electrostatic environment. $1 / f$ noise is of particular concern in sensors, since by its nature it contributes significantly to the degradation of the sensor stability and can result in sensor drift. In [6], the authors study noise in individual SWNT devices, and show that $1 / f$ noise is a significant contributor to the overall noise in the device. They argue that the source of this noise is most likely the trapping and de-trapping of charges from surroundings of the CNT, which can lead to variations in surface potential as well as the number of carriers in the channel. Their results also show that the noise is highest in the subthreshold region of the transfer characteristics. However, signal-to-noise ratio, (and therefore, the sensitivity) rather than just noise needs to be investigated in detail as a figure of merit for sensors. Helbling et al. [26] have investigated the noise in piezoresistive CNFET transducers, and found that the signal-to-noise ratio is maximized in the off-state of small-gap semiconducting CNTs. Mattmann [27] investigated noise in the threshold voltage of CNFETs, as this is typically the sensor output signal. It is found that this also follows a $1 / f$ behavior (Fig. 4).

Furthermore, Collins et al. [24] investigated the noise in many-tube devices, and found that the noise was also high in SWNT networks and mats. Thus, independent of the device configuration and measurement technique, CNT devices are prone 
to $1 / f$ noise, which will have to be studied and considered in their applications in sensors.

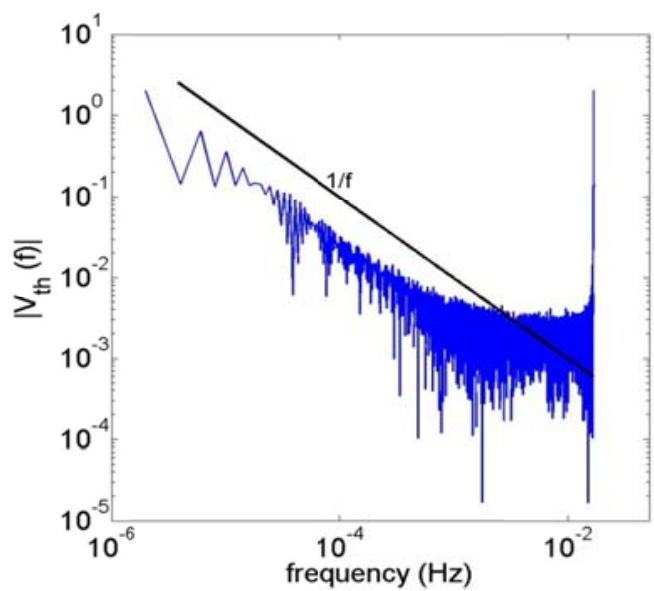

Fig. 4: The threshold voltage noise of a passivated CNFET as a function of frequency, following the technique defined in [27]. A $1 / f$ slope is shown for reference.

\section{Conclusion}

Single-walled carbon nanotube based devices show great potential in ultra-low power chemical sensors due to room temperature operation and high sensitivity to the environment. Novel electrical measurement techniques have been proposed to overcome the problem of hysteresis. Routes to improving selectivity through functionalization have been demonstrated. However, questions about sensing mechanisms and high $1 / f$ noise in these devices remain and will have to be investigated before CNT-based sensors can be applied practically.

\section{Acknowledgements}

The authors are indebted to funding from the European Union FP7 project Technotubes. Support from the FIRST and FIRST-CLA labs at ETH is also gratefully acknowledged.

\section{References}

[1] J. Kong, Franklin N.R., C. Zhou, M. G. Chapline, S. Peng, K. Cho, H. Dai, Science 287, 622-625 (2000) doi: 10.1126/science.287.5453.622

[2] H. Dai, Accounts of Chemical Research 35, 1035 1044 (2002) doi: 10. 1021/ar0101640

[3] A. Jungen, C. Stampfer, J. Hoetzel, V. M. Bright, and C. Hierold, Sensors And Actuators A-Physical, vol. 130, 588-594 (2006) doi:10.1016/j.sna.2005.12.019

[4] T. Helbling, C. Hierold, L. Durrer, C. Roman, R. Pohle, M. Fleischer, physical status solidi (b), vol. 245, 23262330 (2008) doi:10.1002/pssb.200879599

[5] I. Heller, A. M. Janssens, J. Mannik, E. D. Minot, S. G. Lemay, C. Dekker, Nano Letters vol.8 (2), 591-595 (2008) doi: $10.1021 /$ nl072996i

[6] Y. M. Lin, J. Appenzeller, Z. Chen, P. Avouris, Physica E vol. 37, 72-77 (2007) doi:10.1016/j.physe.2006.07.008

[7] T. Helbling, R. Pohle, L. Durrer, C. Stampfer, C. Roman, A. Jungen, M. Fleischer, C. Hierold, Sensors and Actuators B 132, 491-497

(2008);doi:10.1016/j.snb.2007.11.036

[8] W. Kim, A. Javey, O. Vermesh, Q. Wang, Y. Li, H. Dai, Nano Letters vol. 3 (2), 193-198 (2003); doi:10.1021/nl0259232

[9] H. Lin, S. Tiwari, Applied Physics Letters 89, 073507 (2006); doi:10.1063/1.2337104

[10] M. S. Fuhrer, B. M. Kim, T. Dürkop, T. Brintlinger, Nano Letters, vol. 2 (7), 755-759 (2002); doi:10.1021/jp074692q

[11] M. Mattmann, T. Helbling, L. Durrer, C. Roman, C. Hierold, R. Pohle, M. Fleischer, Applied Physics Letters 94, 183502 (2009); doi:10.1063/1.3125259

[12] M. Mattmann, D. Bechstein, C. Roman, K. Chikkadi, C. Hierold, Applied Physics Letters 97, 153103 (2010); doi:10.1063/1.3499363

[13] M. Mattmann, C. Roman, T. Helbling, D. Bechstein, L. Durrer, R. Pohle, ;. Fleischer, C. Hierold, Nanotechnology vol. 21, 185501 (2010); doi:10.1088/0957-4484/21/18/185501

[14] M. Mattmann, D. Bechstein, C. Roman, K. Chikkadi, T. Helbling, L. Durrer, R. Pohle, M. Fleischer, C. Hierold, Procedia engineering 5, 1119-1122 (2010); doi:10.1016/j.proeng.2010.09.307

[15] M. Muoth, T. Helbling, L. Durrer, S.-W. Lee, C. Roman, and C. Hierold, Nature Nanotechnology 5, 589-592 (2010); doi:10.1038/nnano.2010.129

[16] M. Muoth, C. Hierold, Proceedings of the $25^{\text {th }}$ IEEE MEMS 2012, 1352-1355 doi:10.1109/MEMSYS.2012.6170417

[17] J. Li, Y. Lu, Q. Ye, M. Cinke, J. Han, M. Meyyappan, Nano Letters, vol. 3 (7), 929-933 (2003); doi:10.1021/nl034220x

[18] Y. W. Chang, J. S. Oh, S. W. Yoo, H. H. Choi, K. H. Yoo, Nanotechnology vol. 18 (43), 435504 (2007); doi:10.1088/0957-4484/18/43/435504

[19] N. Peng, Q. Zhang, C. L. Chow, O. K. Tan, N. Marzari, Nano Letters vol. 9 (4), 1626-1630 (2009); doi:10.1021/nl803930w

[20] P. Bondavalli, P. Legagneux, D. Pribat, Sensors and Actuators B, vol. 140 (1), 304-318 (2009); doi:10.1016/j.snb.2009.04.025

[21] A. S. Khojin, F. K. Araghii, M. A. Kuroda, K. Y. Lin, J. P. Leburton, R. I. Masel, ACS Nano, vol. 5 (1), 153158 (2011); doi:10.1021/nn101995f

[22] J. Kong, M. G. Chapline, H. Dai, Advanced Materials, vol. 13 (18), 1384-1386 (2001); doi:10.1002/15214095(200109)13:18<1384::AID-ADMA1384>3.0.CO;28

[23] P. Qi, O. Vermesh, M. Grecu, A. Javey, Q. Wang, H. Dai, Nano Letters, vol. 3 (3), 347-351 (2003); doi: 10.1021/nl034010k

[24] P. G. Collins, M. S. Fuhrer, A. Zettl, Applied Physics Letters 76 (7), 894-896 (2000); doi:10.1063/1.125621

[25] J. Appenzeller, Y. M. Lin, J. Knoch, Z. Chen, P. Avouris, IEEE Transactions on Nanotechnology, vol. 6(3), pp. 368- 373 (2007); doi:10.1109/TNANO.2007.892052

[26] T. Helbling, C. Roman, C. Hierold, Nano Letters vol. 10 (9), pp. 3350-3354 (2010); doi:10.1021/nl101031e

[27] Mattmann M., Scientific reports on Micro and Nanosystems vol. 12, PhD Dissertation ETH Zurich No. 19724, 2011. doi: 10.3929/ethz-a-006679957 